\title{
Reconstruction Method for Atom Probe Tomography by Using Field Emission Microscopy*
}

\author{
Yun Kim, ${ }^{\dagger}$ Tsuyoshi Yukawa, and Daichi Shirakura \\ Institute of Industrial Science, The University of Tokyo, \\ Komaba 4-6-1, Meguro-ku, Tokyo 153-8505, Japan \\ Masato Morita \\ Graduate School of Electrical Engineering and Electronics, \\ Kogakuin University, Nakano 2665-1, Hachioji-shi, Tokyo 192-0015, Japan \\ Masanori Owari \\ Institute of Industrial Science, The University of Tokyo, \\ Komaba 4-6-1, Meguro-ku, Tokyo 153-8505, Japan, and \\ Environmental Science Center, The University of Tokyo, \\ Hongo 7-3-1, Bunkyo-ku, Tokyo 113-0033, Japan \\ (Received 8 December 2015; Accepted 23 April 2016; Published 2 July 2016)
}

\begin{abstract}
Atom probe tomography (APT) is a three-dimensional (3D) analysis technique with atomic resolution in materials science and engineering. The ionized atoms are sequentially released from the surface of the sample with needle shape by applying high voltage and are detected by the position-sensitive detector. The original arrangement is reconstructed using reconstruction parameters such as the image compression factor and the field factor from recorded data during measurement. It is crucial to the integrity of the reconstruction to estimate the reconstruction parameters as accurately as possible. However, it is difficult to determine the reconstruction parameters accurately due to sequential change of the shape of the sample during measurement. In this study, by using FEM we propose a new reconstruction method that the reconstruction parameters can be determined even during the measurement. [DOI: $10.1380 /$ ejssnt.2016.189]
\end{abstract}

Keywords: Field emission microscopy; Field emission; Field evaporation; Tungsten

\section{INTRODUCTION}

Atom probe tomography (APT) is a three-dimensional (3D) analysis technique with atomic resolution in materials science and engineering [1]. This technique is based on the field evaporation. When high positive voltage is applied to a needle-shape sample whose radius of curvature is less than $100 \mathrm{~nm}$, the strong electric field $\left(\sim 100 \mathrm{Vnm}^{-1}\right)$ is generated. When the electric field is sufficiently strong, the atom is released from the surface of the sample while one of its electrons is tunneled into the surface. The ionized atom is accelerated and reaches the position-sensitive detector. The original position of ionized atom is calculated from the detected position and sequence, and identification of element is determined from the time-of-flight of ion.

The original arrangements of atoms are reconstructed from acquired data using reconstruction parameters such as the image compression factor, the shank angle and the field factor, and finally the atomic arrangement is expressed as 3D image. It is crucial to the integrity of the reconstruction to estimate the reconstruction parameters as accurately as possible for accurate analysis. However, it is difficult to determine the reconstruction parameters accurately because of sequential change of the sample shape during measurement. For accurate reconstruction, the

\footnotetext{
* This paper was presented at the 10th International Symposium on Atomic Level Characterizations for New Materials and Devices '15, Shimane Prefectural Convention Center (Kunibiki Messe), Matsue, Japan, 25-30 October, 2015.

$\dagger$ Corresponding author: kyyk1001@iis.u-tokyo.ac.jp
}

shape of the sample is observed by using Transmission Electron Microscopy (TEM). However, the observation of the sample shape is possible only before and after APT measurement. In the other words, it is difficult to observe the change of the sample shape during measurement. In the case of using pulses laser for field evaporation, that has especially influence on the accuracy of reconstruction. By direction of the laser irradiation, the irradiation side and the shadow side are generated at the sample surface. It is known that atoms are more easily ionized at irradiation side than at shadow side for the beginning of measurement [2]. Then, the radius of curvature of the irradiation side is larger than that of the shadow side. Because the electric field is reverse correlation to the radius of curvature of the sample, the rate of field evaporation becomes higher at the shadow side. In eventually, the rate of field evaporation becomes a steady state which the rate of the field evaporation is the same at the irradiation side and at the shadow side [3]. Therefore, the sample shape is changed during measurement. For accurate reconstruction, it is necessary that the change of the sample shape is observed during measurement.

Field Emission Microscopy (FEM) is based on the field emission of electrons. When negative high voltage is applied to the sample, the strong electric field is generated. Then the electrons at the sample surface are moved from the sample surface to vacuum [4]. The emitted electrons are detected by phosphor-screen. The electrons are more easily tunneled at the position where electric field is stronger. When more electrons are detected at one position compared to other position, it means that electrons are more easily tunneled to vacuum at this position. In other words, the position of the highest electric field at 
the surface of the sample may be estimated from the intensities of electrons at each position. In the case of APT measurement, the atom is more easily ionized at the position where electric field is stronger than other positions. Therefore, we supposed that the position where intensities of emitted electrons were high is also high intensities of emitted ions. If this hypothesis was true, we could know the position of the highest ion intensities from FEM data.

In current work, we studied relevance between ion and electron emission intensities at each position.

\section{EXPERIMENTAL}

\section{A. Sample Preparation}

In this study, we used $\mathrm{W}$ as samples for measurements of FEM and APT measurement. The samples were processed to $50 \sim 100 \mathrm{~nm}$ in radius curvature by electrolytic polishing and using Focused Ion Beam (FIB). The radii of the samples were measured by TEM.

\section{B. Experimental Method}

To confirm the correlativity between ions and electrons emission intensities at each position, we alternately measured FEM and APT by using developed APT in our laboratory with laser wavelength of $532 \mathrm{~nm}$, laser power of $2.5 \mathrm{~nJ} /$ pulse, laser pulse width of $300 \mathrm{fs}$ and pulse frequency of $2.5 \mathrm{kHz}$. The sample temperature was room temperature or $60 \mathrm{~K}$.

First, we measured the electron intensity at each position by applied negative voltage and pulse laser until the counts of detected electron were about 300,000 . Then, the sample was applied positive voltage for measurement of the ion intensity at each position until the detected $\mathrm{W} 2+$ and $\mathrm{W} 3+$ ions were about 150,000 counts, we repeated these process 7 times for each sample. The each surface of tip which was formed by each FEM and APT measurement, we confined the surface. Therefore, the surface 1 was more out surface than the surface 7 . The experimental condition of FEM measurement was the same as that of APT measurement.

The intensities of ions and electrons were calculated by integrating whole region at each $\mathrm{X}$ and $\mathrm{Y}$ axis. The intensities density at each position was calculated on the basis of the size of at each position.

In the case of FEM, the electrons were emitted at one position and the flight paths of the emitted electrons were respectively different because the electrons had been difference kinetic energies. Therefore it is difficult to accurately estimate the position of the emitted electrons at surface of the sample. In this study, we calculated the position of the emitted electrons in the same condition as ions.

An overall scheme of experimental method and the direction of laser irradiation were shown in Fig. 1. The detector was placed in the vertical direction of the sample and the distance was $10 \mathrm{~cm}$ between the tip of the sample and the detector. For the correction of flight time of ions and electron, the rotation $(\alpha)$ of Y-axis of the de- (a)

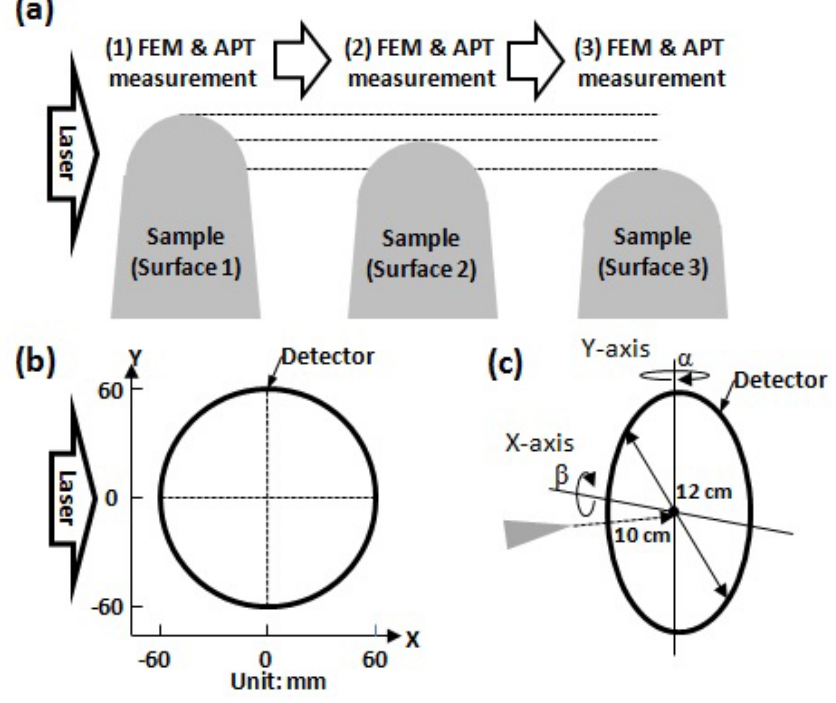

FIG. 1. (a) Schematic of experimental method. (b) Direction of laser irradiation. (c) The scheme of the sample and the detector.
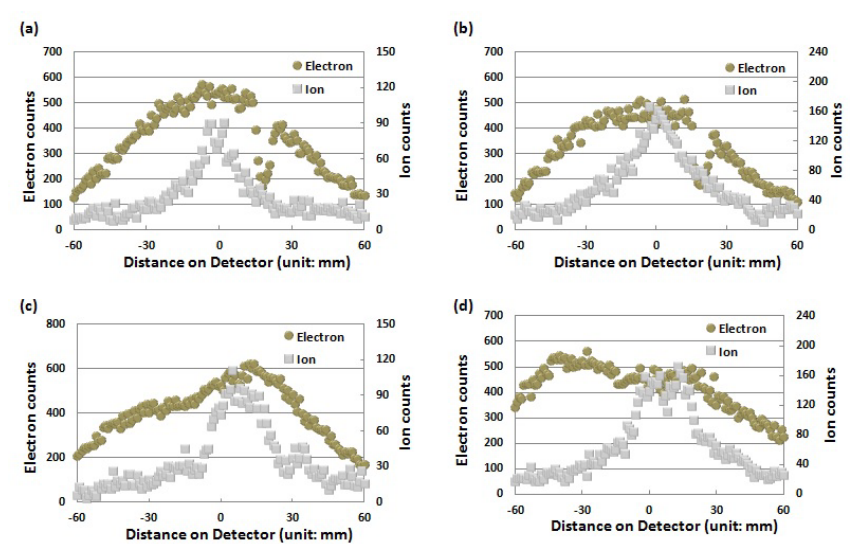

FIG. 2. The counts of emitted ions and electrons at each position (room temperature). (a) Surface 1, Y axis, (b) Surface 3, $\mathrm{Y}$ axis, (c) Surface 1, $\mathrm{X}$ axis, (d) Surface 3, $\mathrm{X}$ axis.

tector was 1.4 degree and the $\operatorname{rotation}(\beta)$ of $\mathrm{X}$-axis was 1.0 degree (Fig. 1(c)).

\section{RESULTS AND DISCUSSIONS}

\section{A. Ion and Electron Emission Intensities at Each Position at Room Temperature}

The intensities of emitted ions and electrons at each position were shown in Fig. 2. The intensity at the Xaxis or at $\mathrm{Y}$-axis was representation of the intensities of all areas in the directions of the $\mathrm{X}$-axis or $\mathrm{Y}$-axis respectively (about 10 counts $/ \mathrm{mm}^{2}$ ).

In the case of $\mathrm{Y}$ axis at Surface 1, the intensities of electrons were the strongest at the nearby center of the detector. The intensities of ions were also the strongest at nearby center of the detector (Fig. 2(a)). In the case Sur- 

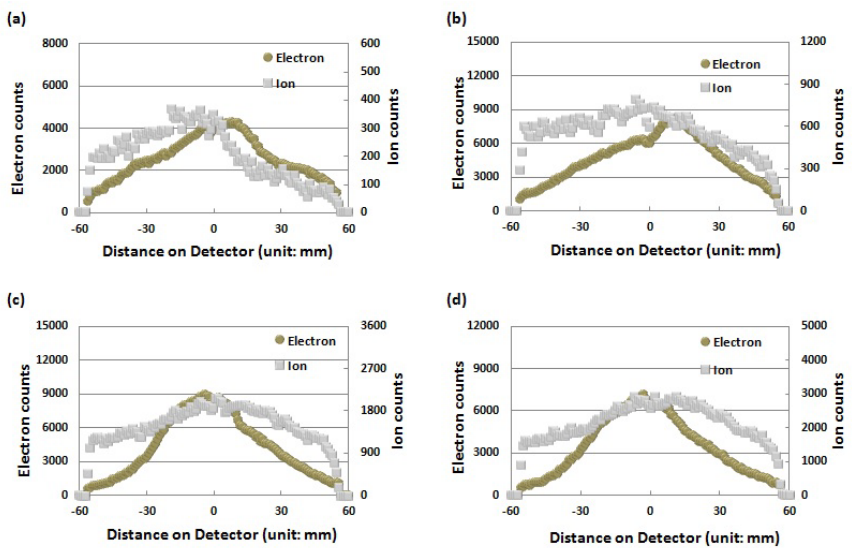

FIG. 3. The counts of emitted ion and electron at each position (60 K, X axis). (a) Surface 1, (b) Surface 3, (c) Surface 5, (d) Surface 7.

face 3 , the inside of the sample, the highest intensity position of ions was also close to that of electrons (Fig. 2(b)). In the case of $\mathrm{X}$ axis at Surface 1, the highest intensity position of ions was close to that of electrons (Fig. 2(c)). However, the highest intensity position of electrons was shifted to laser irradiation direction (Fig. 2(d)) at Surface 3 although the highest intensity position of ions was not moved. One possible explanation of the shift of strongly emitted position of electrons was thermal effect by irradiated laser. The shadow side and the irradiation side were formed in the sample because the laser was irradiated from one-direction (Fig. 1(b)). It was known that the temperature of the irradiation side was higher than that of shadow side by the irradiated laser $[5,6]$. The potential energy of electrons at the irradiation side were raised by the irradiated laser, the electrons might be easily emitted at the irradiation side than the shadow side. Particularly because the laser was irradiated along $\mathrm{X}$ axis direction, difference of temperature at $\mathrm{X}$ axis was greater than $\mathrm{Y}$ axis. Therefore, the shift of position where electrons were strongly emitted in $\mathrm{X}$ axis might be greater than $\mathrm{Y}$ axis at room temperature during measurement.

\section{B. Ion and Electron Emission Intensities at Each Position at $60 \mathrm{~K}$}

We measured the cooled sample to check the thermal effect by irradiated laser. The temperature of the sample was $60 \mathrm{~K}$ during measurement. The experimental method was the same as the experiment at room temperature. The results were shown in Figs. 3 and 4 . In the case of $\mathrm{X}$ axis, the highest intensity position of ions was close to that of electrons at the outmost surface (surface 1) and the inside surface (surface 7) (Figs. 3(a)-(d)).

However the result of $\mathrm{Y}$ axis was different that of $\mathrm{X}$ axis. The intensities of electrons were low at the center of detector and the strong intensity positions were the edges of detector (Fig. 4). Furthermore, the distance between the strong intensity positions became larger at inside of the sample (Figs. 4(a)-(d)). In the case of ion, it was shown that the nearby center positions were higher than
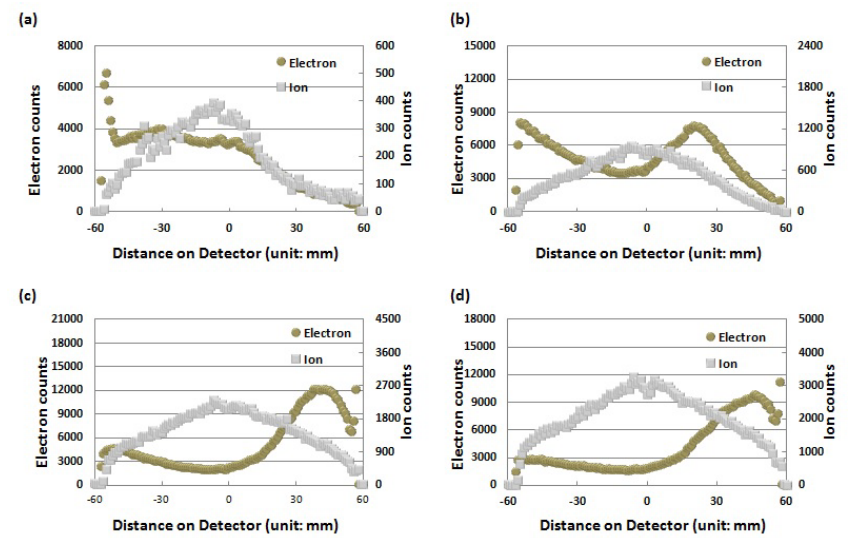

FIG. 4. The counts of emitted ion and electron at each position (60 K, Y axis). (a) Surface 1, (b) Surface 3, (c) Surface 5, (d) Surface 7 .

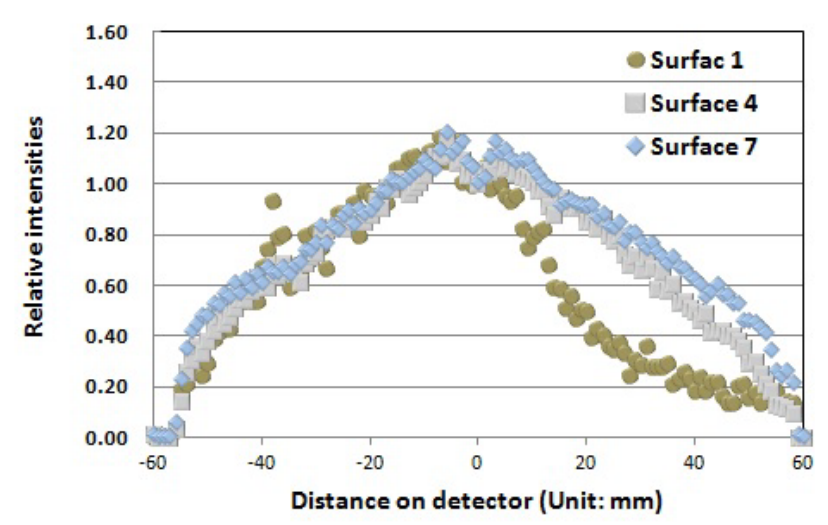

FIG. 5. The relative intensities of ions at $\mathrm{Y}$ axis.

other positions at all surfaces (Surface 1-7). One possible explanation of this result was the change of the sample shape. The laser was irradiated from perpendicular to $\mathrm{Y}$ axis. The shadow and the irradiation side were not generated in $\mathrm{Y}$ axis. However, the center of the sample was more affected the laser than the edge because the sample was needle-like shape. Therefore the rate of field evaporation at the center of the sample was higher than at the edge of the sample. According to difference of field evaporation rate between the center and the edge, the curvature of radius of the center became larger than that of the edge. Then the electric field at edge became stronger than that of the center. Therefore, the electrons were more easily emitted at edge. In the case of ion at $\mathrm{Y}$ axis, the distribution of ions intensities did not show the significant change compared to that of electrons because the field evaporation was required stronger electric filed compared to field emission. Therefore, the ions were more emitted at the center.

Figure 5 was shown the comparison of ion intensity distributions along $\mathrm{Y}$ axis. The relative intensities were calculated based on the intensity of the center. The distribution of the intensity at Surface 7 was wider than that of Surface 1. It means that the sample shape was changed. 


\section{CONCLUSIONS}

In this study, we studied relevance between ion and electron emission intensities at each position. It was observed that the intensities of electrons were shifted related to the change of the sample shape. It means that the change of the sample shape during APT measurement was estimated from FEM data which was shown the intensities of electrons at each position. It was suggested relative ion emission intensities at each position might be estimated at each position by using FEM.
[1] M. K. Miller, Atom Probe Tomography Analysis at Atomic Level (Kluwer Acandmic/Plenum Publishers, New York, 2000).

[2] G. Sha et al., Appl. Phys. Lett. 92, 043503 (2008).

[3] M. N. Bachhav et al., Appl. Phys. Lett. 99, 084101 (2011).
[4] R. Gomer, Surf. Sci. 299/300, 129 (1994).

[5] B. Gault, F. Vurpillot, A. Bostel, A. Menand, and B. Deconihout, Appl. Phys. Lett. 86, 094101 (2005).

[6] M. Tsukada, H. Tamura, K. P. McKenna, A. L. Shluger, Y. M. Chen, T. Ohkubo, and K. Hono, Ultramicroscopy 111, 567 (2011). 